Bull. Korean Math. Soc. 45 (2008), No. 4, pp. 645-650

\title{
ORBITAL SHADOWING PROPERTY
}

\author{
Bahman Honary and Alireza Zamani Bahabadi
}

\begin{abstract}
Let $M$ be a generalized homogeneous compact space, and let $Z(M)$ denotes the space of homeomorphisms of $M$ with the $C^{0}$ topology. In this paper, we show that if the interior of the set of weak stable homeomorphisms on $M$ is not empty then for any open subset $W$ of $Z(M)$ containing only weak stable homeomorphisms the orbital shadowing property is generic in $W$.
\end{abstract}

\section{Introduction}

The concept of shadowing is investigated by many authors (see e.g. $[2,4$, 8]). In [3] Corless and Pilyugin proved that weak shadowing is a $C^{0}$ generic property for discrete dynamical systems of a compact smooth manifold $M$. Subsequently, Pilyugin and Plamenevskaya [9] improved this result by showing $C^{0}$ genericity of the shadowing property. Both proofs given in [3] and [9] required that $M$ be a $C^{\infty}$ smooth manifold. Mazur in [7] showed that for $C^{0}$ genericity of weak shadowing neither the differential structure on $M$, nor even being a manifold is a crucial assumption, but what matters is a generalized version of a topological property called homogeneity. Koscielniak and Mazur in [5] have given a proof for $C^{0}$ genericity of periodic orbital shadowing on a compact topological manifold of dimension at least 2 . In this note we show that if the space $M$ is generalized homogeneous and has no isolated points, then in an open subset of $Z(M)$ the orbital shadowing property is generic.

\section{Notations}

Let $(M, d)$ be a compact metric space and let $f: M \rightarrow M$ be a homeomorphism (a discrete dynamical system on $M$ ). A sequence $\left\{x_{n}\right\}_{n \in \mathbb{Z}}$ is called an orbit of $f$, denote by $o(x, f)$, if for each $n \in \mathbb{Z}, x_{n+1}=f\left(x_{n}\right)$ and we call it a $\delta$-pseudo-orbit of $f$ if,

$$
d\left(f\left(x_{n}\right), x_{n+1}\right) \leq \delta, \forall n \in \mathbb{Z} .
$$

The homeomorphism $f$ is said to have the weak shadowing property if for each $\epsilon>0$ there exists $\delta>0$ such that for any $\delta$-pseudo-orbit $\left\{x_{n}\right\}_{n \in \mathbb{Z}}$ of $f$ we

Received June 24, 2007; Revised August 22, 2008.

2000 Mathematics Subject Classification. Primary 54H20; Secondary 54F99.

Key words and phrases. orbital shadowing property, $\delta$-pseudo-orbit, shadowing property. 
can find a point $y \in M$ such that $\left\{x_{n}\right\}_{n \in \mathbb{Z}} \subset N_{\epsilon}(o(y, f))$, where $N_{\epsilon}(S)$ is the $\epsilon$-neighborhood of the set $S \subset M$.

A system $f$ is said to have the orbital shadowing property if for each $\epsilon>0$ there exists $\delta>0$ such that for any $\delta$-pseudo-orbit $\left\{x_{n}\right\}_{n \in \mathbb{Z}}$ of $f$ we can find a point $y \in M$ with the property that $\left\{x_{n}\right\}_{n \in \mathbb{Z}} \subset N_{\epsilon}(o(y, f))$ and $o(y, f) \subset$ $N_{\epsilon}\left(\left\{x_{n}\right\}_{n \in \mathbb{Z}}\right)$. We denoted the set of all homeomorphisms of $M$ by $Z(M)$. Introduce in $Z(M)$ the complete metric

$$
d_{0}(f, g)=\max \left\{\max _{x \in M} d(f(x), g(x)), \max _{x \in M} d\left(f^{-1}(x), g^{-1}(x)\right)\right\},
$$

which generates the $C^{0}$ topology.

The space $M$ is said to be generalized homogeneous if for every $\epsilon>0$ there exists $\delta>0$ such that if $\left\{x_{1}, \ldots, x_{n}\right\},\left\{y_{1}, \ldots, y_{n}\right\} \subset M$ is a pair of sets of mutually disjoint elements satisfying $d\left(x_{i}, y_{i}\right) \leqslant \delta, i \in\{1, \ldots, n\}$, then there exists $h \in Z(M)$ satisfying $d_{0}\left(h, i d_{M}\right) \leqslant \epsilon$ and $h\left(x_{i}\right)=y_{i}, i \in\{1, \ldots, n\}$. Such a $\delta$ is called an $\epsilon$-modulus of homogeneity of $M$. We say that $x \in M$ is a weak stable point for $f$ if for any $\epsilon>0$ there is $\delta>0$ and positive integer $N$ such that $o(z, f) \subset N_{\epsilon}\left(\left\{f^{i}(z): i=-N, \ldots, N\right\}\right)$ for every $z \in M$ with $d(x, z)<\delta$. We say that $f$ is weak stable if every point of $M$ is a weak stable point for $f$.

A property $P$ is said to be generic for elements of a topological space $M$ if the set of all $x \in M$ satisfying $P$ is residual, i.e., it includes a countable intersection of open and dense subsets of $M$.

\section{Results}

Proposition 1. Let $f$ be a homeomorphism on a compact metric space $M$. Then the set of weak stable points is residual in $M$.

Proof. Fix an $\epsilon>0$. Let $U=\left\{U_{i}: i=1, \ldots, k\right\}$ be a finite covering of $M$ by open sets with diameter less than $\frac{\epsilon}{2}$. Put $K=\{1,2, \ldots, k\}$. For each $x \in M$, choose a subset $L_{x}$ of $K$ satisfying the following conditions:

$$
\begin{gathered}
o(x, f) \subset \cup\left\{U_{i}: i \in L_{x}\right\} \\
o(x, f) \cap U_{i} \neq \phi \text { for all } i \in L_{x} .
\end{gathered}
$$

Let $A_{\epsilon}$ be the set of all $x \in M$ such that there is $\delta_{x}$ and positive integer $N_{x}$ such that $o(z, f) \subset N_{\epsilon}\left(\left\{f^{i}(z): i=-N_{x}, \ldots, N_{x}\right\}\right)$ for every $z \in M$ with $d(x, z)<\delta_{x}$. We claim that $A_{\epsilon}$ is open and dense in $M$. Clearly $A_{\epsilon}$ is open. To see that $A_{\epsilon}$ is dense, let $x \in M$ be arbitrary. We can find a positive integer $T$ such that

$$
\left\{f^{i}(x): i=-T, \ldots, T\right\} \cap U_{j} \neq \phi \text { for all } j \in L_{x} .
$$

Hence $o(x, f) \subset N_{\epsilon}\left(\left\{f^{i}(x): i=-T, \ldots, T\right\}\right)$. Choose $\delta>0$ such that

$$
d\left(f^{i}(x), f^{i}(z)\right)<\frac{\epsilon}{2} \text { for all } i=-T,-T+1, \ldots, T
$$

for every $z \in M$ with $d(x, z)<\delta$. Suppose that $x \notin A_{\epsilon}$. Given any $\zeta$ with $0<\zeta<\delta$ there is $x_{1} \in N_{\zeta}(x)$ such that $f^{T_{1}}\left(x_{1}\right) \notin N_{\epsilon}\left(\left\{f^{i}(x): i=-T, \ldots, T\right\}\right)$ 
for some $T_{1}$ with $\left|T_{1}\right|>T$. If $f^{T_{1}}\left(x_{1}\right) \in N_{\frac{\epsilon}{2}}\left(\left\{f^{i}(x): i=-T, \ldots, T\right\}\right)$, then we have

$$
d\left(f^{T_{1}}\left(x_{1}\right), f^{i}\left(x_{1}\right)\right) \leq d\left(f^{T_{1}}\left(x_{1}\right), f^{i}(x)\right)+d\left(f^{i}(x), f^{i}\left(x_{1}\right)\right)<\frac{\epsilon}{2}+\frac{\epsilon}{2}=\epsilon
$$

for some $i \in\{-T, \ldots, T\}$. This is a contradiction. Thus

$$
f^{T_{1}}\left(x_{1}\right) \notin N_{\frac{\epsilon}{2}}\left(\left\{f^{i}(x): i=-T, \ldots, T\right\}\right) .
$$

Since $f_{1}^{T}\left(x_{1}\right) \notin U_{j}$ for all $j \in L_{x}$, there is $j \in K-L_{x}$ such that $f^{T_{1}}\left(x_{1}\right) \in U_{j}$. Thus $L_{x}$ is a proper subset of $L_{x_{1}}$. We can find $T_{2} \geq\left|T_{1}\right|$ such that

$$
o\left(x_{1}, f\right) \subset N_{\epsilon}\left(\left\{f^{i}\left(x_{1}\right): i=-T_{2}, \ldots, T_{2}\right\}\right) .
$$

Choose $\delta_{1}>0$ such that $d\left(f^{i}\left(x_{1}\right), f^{i}(z)\right)<\frac{\epsilon}{2}$ for all $i \in\left\{-T_{2},-T_{2}+1, \ldots, T_{2}\right\}$ for every $z \in M$ with $d\left(x_{1}, z\right)<\delta_{1}$. If $x_{1} \in A_{\epsilon}$ we are done, otherwise there is $x_{2} \in N_{\xi}\left(X_{1}\right) \subset N_{\zeta}(x)$ such that

$$
f^{T_{3}}\left(x_{2}\right) \notin N_{\epsilon}\left(\left\{f^{i}\left(x_{2}\right): i=-T_{2}, \ldots, T_{2}\right\}\right)
$$

for some $T_{3}$ with $\left|T_{3}\right|>T_{2}$, where $\xi=\min \left(\zeta, \delta_{1}\right)$. Since

$$
f^{T_{3}}\left(x_{2}\right) \notin N_{\frac{\epsilon}{2}}\left(\left\{f^{i}\left(x_{1}\right): i=-T_{2}, \ldots, T_{2}\right\}\right),
$$

$f_{3}^{T}\left(x_{2}\right) \notin U_{j}$ for all $j \in L_{x}$. There is $j \in K-L_{x_{1}}$ such that $f^{T_{3}}\left(x_{2}\right) \in U_{j}$. Thus $L_{x_{1}}$ is a proper subset of $L_{x_{2}}$. By continuing this process, since $K$ is finite, we can find $x^{\prime} \in N_{\xi}(x)$ such that $L_{x^{\prime}}=K$. Then $x^{\prime} \in A_{\epsilon}$. Thus $A_{\epsilon}$ is dense in $M$. Now let $R=\bigcap_{n=1}^{\infty} A_{\frac{1}{n}}$ then $R$ is a residual subset of $M$ consisting of weak stable points.

A homeomorphism $f: M \longrightarrow M$ is called minimal if $f(A)=A$, A closed, implies either $A=M$ or $A=\phi$. It is easy to see that $f$ is minimal if and only if $\overline{o(x, f)}=M$ for each $x \in M$.

Proposition 2. Let $f$ be a homeomorphism on a compact metric space $M$. If $f$ is minimal, then $f$ is weak stable.

Proof. Let $x \in M$ and $\epsilon>0$ be arbitrary. Let $U=\left\{U_{i}: i=1, \ldots, k\right\}$ be a finite covering of $M$ by open sets with diameter less than $\frac{\epsilon}{2}$. Since $\overline{o(x, f)}=M$, there are $n_{1}, n_{2}, \ldots, n_{k} \in \mathbb{Z}$, such that $f^{n_{i}}(x) \in U_{i}$ for $i=1,2, \ldots, k$. Let

$$
N=\max \left\{\left|n_{i}\right|: 1 \leq i \leq k\right\} .
$$

There exists $\delta>0$ such that if $d(x, y)<\delta$, then $d\left(f^{i}(x), f^{i}(y)\right)<\frac{\epsilon}{2}$ for all $-N \leq i \leq N$. Let $y \in N_{\delta}(x)$. Given any $n \in \mathbb{Z}$, since $f^{n}(y) \in M=\bigcup_{i=1}^{k} U_{i}$, $f^{n}(y) \in U_{i}$ for some $i=1, \ldots, k$. But $f^{n_{i}}(x) \in U_{i}$ so $d\left(f^{n}(y), f^{n_{i}}(x)\right) \leq$ $\operatorname{diam}\left(U_{i}\right)<\frac{\epsilon}{2}$. Since $d(x, y)<\delta$ and $-N \leq n_{i} \leq N$ we have $d\left(f^{n_{i}}(x), f^{n_{i}}(y)\right)<$ $\frac{\epsilon}{2}$. Thus we have

$$
d\left(f^{n}(y), f^{n_{i}}(y)\right) \leq d\left(f^{n}(y), f^{n_{i}}(x)\right)+d\left(f^{n_{i}}(x), f^{n_{i}}(y)\right)<\frac{\epsilon}{2}+\frac{\epsilon}{2}=\epsilon .
$$

Hence $o(y, f) \subset N_{\epsilon}\left(\left\{f^{i}(y): i=-N, \ldots, N\right)\right.$. This shows that $x$ is an weak stable point and $f$ is an weak stable homeomorphism. 
We denote the set of all weak stable homeomorphisms by WSH.

Theorem 1. Let $\operatorname{int}(W S H) \neq \phi$ and let $W$ be an open subset of $Z(M)$ containing only weak stable homeomorphisms. Then there is a residual subset $R_{1}$ of $W$ such that for each $f \in R_{1}$ and $\epsilon>0$, there is a neighborhood $U_{f}$ of $f$ and positive integer $N_{f}$ such that $o(x, g) \subset \bigcup_{i=-N_{f}}^{N_{f}} \overline{N_{\epsilon}\left(g^{i}(x)\right)}$ for every $g \in U_{f}$ and $x \in M$.

To prove this theorem we need the following two lemmas.

Lemma 1. Let $\epsilon>0$ be arbitrary. Then the function $\psi_{\epsilon}: W \longrightarrow \mathbb{N}$; defined by

$$
\psi_{\epsilon}(f)=N_{f},
$$

where

$$
N_{f}=\min \left\{N \in \mathbb{N}: o(x, f) \subset \bigcup_{i=-N}^{N} \overline{N_{\epsilon}\left(f^{i}(x)\right)} \forall x \in M\right\},
$$

is lower semi-continuous.

Proof. For $f \in W$ there is $x_{0} \in M$ such that $o\left(x_{0}, f\right) \nsubseteq \bigcup_{i=-N_{f}+1}^{N_{f}-1} \overline{N_{\epsilon}\left(f^{i}\left(x_{0}\right)\right)}$. So $f^{k}\left(x_{0}\right) \notin \bigcup_{i=-N_{f}+1}^{N_{f}-1} \overline{N_{\epsilon}\left(f^{i}\left(x_{0}\right)\right)}$ for some $k \in \mathbb{Z}$ with $|k| \geq N_{f}$. Choose $\epsilon^{\prime}>0$ such that

$$
d\left(f^{N_{f}}\left(x_{0}\right), f^{l}\left(x_{0}\right)\right) \geq \epsilon+\epsilon^{\prime},-N_{f}+1 \leq l \leq N_{f}-1 .
$$

Choose a neighborhood $U_{f}$ of $f$ such that $d\left(f^{i}(x), g^{i}(x)\right)<\frac{\epsilon^{\prime}}{2},|i| \leq k+1$ for each $x \in M$ and $g \in U_{f}$. If $N_{g}<N_{f}$, then $d\left(g^{k}\left(x_{0}\right), g^{l}\left(x_{0}\right)\right)<\epsilon$ for some $-N_{f}+1 \leq l \leq N_{f}-1$. So

$$
\begin{aligned}
d\left(f^{k}\left(x_{0}\right), f^{l}\left(x_{0}\right)\right) & \leq d\left(f^{k}\left(x_{0}\right), g^{k}\left(x_{0}\right)\right)+d\left(g^{k}\left(x_{0}\right), g^{l}\left(x_{0}\right)\right)+d\left(g^{l}\left(x_{0}\right), f^{l}\left(x_{0}\right)\right) \\
& <\frac{\epsilon^{\prime}}{2}+\epsilon+\frac{\epsilon^{\prime}}{2}=\epsilon+\epsilon^{\prime},
\end{aligned}
$$

which contradicts $(*)$. Hence $N_{g} \geq N_{f}$. This complete the proof of the lemma.

Now, we recall a topology lemma, for the proof see [6].

Lemma 2. Let $X$ be a Bair topological space and $\Gamma: X \longrightarrow \mathbb{N}$ be a lower semi-continuous map. Then there exists a residual subset $R$ of $X$ such that $\left.\Gamma\right|_{R}$ is locally constant on each point of $R$.

Proof of Theorem 1. Using lemmas 1 and 2, for any $\epsilon>0$ let $R_{\epsilon}$ be a residual subset of $W$ such that $\psi_{\epsilon}$ is locally constant on $R_{\epsilon}$. Then $R_{1}=\cap\left\{R_{\frac{1}{n}}: n=\right.$ $1,2, \ldots\}$ is the required residual set.

Theorem 2. Let $M$ be a generalized homogeneous space with no isolated point. Then either $\operatorname{int}(W S H)=\phi$, or for every $f \in \operatorname{int}(W S H)$ and every open neighborhood $W$ of $f$ in $\operatorname{int}(W S H)$ the orbital shadowing property is generic in $W$. 
For the proof we need the following lemma from [10].

Lemma 3. If $h$ is upper semi-continuous, then for any $\epsilon>0$ the set of all $x \in X$ such that there exists a neighborhood $U$ of $x$ with the property that $d_{H}(h(x), h(y)) \leq \epsilon$ for all $y \in U$, is open and dense in $X$. Here $d_{H}$ is the Hausdorff metric.

Proof of Theorem 2. Assuming $\operatorname{int}(W S H) \neq \phi$. Let $f \in \operatorname{int}(W S H)$ and $W$ be an open neighborhood of $f$ in $\operatorname{int}(W S H)$. Let $\epsilon>0$ be arbitrary, and $A=\left\{U_{1}, \ldots, U_{k}\right\}$ be a finite covering of $M$ by closed sets with diameter less than $\frac{\epsilon}{2}$. Consider the set $K=\{1,2, \ldots, k\}$ as a compact metric space with discrete metric. Let $C(K)$ be the set of all subset of $\mathrm{K}$, then define the map $\varphi_{\epsilon}: Z(M) \longrightarrow C(C(K))$ by

$$
\begin{gathered}
\varphi_{\epsilon}(f)=\left\{L \subset K: \exists x \in M \text { such that } o(x, f) \subset \bigcup\left\{U_{i}: i \in L\right\},\right. \\
\left.o(x, f) \cap U_{i} \neq \phi \forall i \in L\right\} .
\end{gathered}
$$

The map $\varphi_{\epsilon}$ is upper semi-continuous [1]. Let $R_{\epsilon}$ be the set of all $f \in$ $Z(M)$ such that there exists a neighborhood $U_{f}$ of $f$ with the property that $d_{H}\left(\varphi_{\epsilon}(f), \varphi_{\epsilon}(g)\right) \leq \epsilon$ for every $g \in U_{f}$. The set $R_{\epsilon}$ is open and dense in $Z(M)$ by Lemma 3. Moreover, it is easy to see that the map $\varphi_{\epsilon}$ is locally constant on the set $R_{\epsilon}$ if $\epsilon<1$ that is for any $f \in R_{\epsilon}$ there is a neighborhood $U_{f}$ of $f$ satisfying $\varphi_{\epsilon}(f)=\varphi_{\epsilon}(g)$ for all $g \in U_{f}$. Consider $R_{2}=\bigcap_{n=1}^{\infty} R_{\frac{1}{n}}$ and $R=R_{2} \bigcap R_{1} \bigcap W$, where $R_{1}$ is as in Theorem 1. To complete the proof , it remains to show that the set $R$ has orbital shadowing property. Let $0<\epsilon<1$ be arbitrary and $f \in R$. There is a neighborhood $U_{f}$ of $f$ satisfying $\varphi_{\epsilon}(f)=\varphi_{\epsilon}(g)$ and $\psi_{\epsilon}(f)=\psi_{\epsilon}(g)$ for all $g \in U_{f}$. Choose $\beta>0$ such that $N_{\beta}(f) \subset U_{f}$. Let $\gamma>0$ be a $\beta$-modulus of homogeneity of $M$, and put $0<\delta<\min \left\{\frac{\gamma}{2}, \frac{\epsilon}{2}\right\}$. Fix any $\delta$-pseudo-orbit $y=\left\{y_{n}\right\}_{n \in \mathbb{Z}}$. There exists a positive integer $l \geq N_{f}$ such that $y \subset N_{\epsilon}\left(y_{t}\right)$ where $y_{t}=\left\{y_{n}\right\}_{n=-l}^{l}$. Since $M$ has no isolated point we can easily find a finite $2 \delta$-pseudo-orbit $y_{t}^{\prime}=\left\{y_{n}^{\prime}\right\}_{n=-l}^{l}$ such that $y_{t} \subseteq N_{\epsilon}\left(y_{t}^{\prime}\right)$ and $y_{t}^{\prime} \subseteq N_{\epsilon}\left(y_{t}\right)$ and $y_{i} \neq y_{j}$ for $i \neq j([11])$. Since $d\left(f\left(y_{i}^{\prime}\right), y_{i+1}^{\prime}\right)<2 \delta<\gamma$ there exists $h \in Z(M)$ such that $d_{0}\left(h, i d_{M}\right) \leq \beta$ and $h\left(f\left(y_{i}^{\prime}\right)\right)=y_{i+1}^{\prime}$ for all $i=-l, \ldots, l$. Set $g=h o f$. Then the sequence

$$
o\left(y_{0}^{\prime}, g\right)=\left\{\ldots, g^{-2}\left(y_{-l}^{\prime}\right), g^{-1}\left(y_{-l}^{\prime}\right), y_{-l}^{\prime}, y_{-l+1}^{\prime}, \ldots, y_{l}^{\prime}, g\left(y_{-l}^{\prime}\right), g^{2}\left(y_{-l}^{\prime}\right), \ldots\right\}
$$

is an orbit of $g$. Since $g \in N_{\beta}(f)$ we have $\varphi_{\epsilon}(f)=\varphi_{\epsilon}(g)$ and $\psi_{\epsilon}(f)=\psi_{\epsilon}(g)$. Choose $L \in \varphi_{\epsilon}(g)$ such that $o\left(y_{0}^{\prime}, g\right) \subset \bigcup_{i \in L} U_{i}$ and $o\left(y_{0}^{\prime}, g\right) \cap U_{i} \neq \phi$ for all $i \in L$. But $L \in \varphi_{\epsilon}(f)$, thus there exists $x \in M$ satisfying $o(x, f) \subset \bigcup_{i \in L} U_{i}$ and $o(x, f) \cap U_{i} \neq \phi$ for all $i \in L$. This implies that $y \subseteq N_{3 \epsilon}(o(x, f)), o(x, f) \subset$ $N_{\epsilon}\left(o\left(y_{0}^{\prime}, g\right)\right)$. Since $\psi_{\epsilon}(f)=\psi_{\epsilon}(g)$ we have $o\left(y_{0}^{\prime}, g\right) \subset \bigcup_{n=-N_{f}}^{N_{f}} \overline{N_{\epsilon}\left(y_{n}^{\prime}\right)} \subset$ $\bigcup_{n=-l}^{l} \overline{N_{\epsilon}\left(y_{n}^{\prime}\right)}$ and $y_{t}^{\prime} \subset N_{\epsilon}\left(y_{t}\right) \subset N_{\epsilon}(y)$. Hence we get $o(x, f) \subset N_{3 \epsilon}(y)$. This complete the proof of Theorem 2 .

As Mazur has shown in [7] the spaces (i), (ii) and (iii) in the following corollary are homogeneous. Thus using Theorem 2 we have: 
Corollary. If the space $M$ is one of the followings:

(i) a topological manifold with boundary ( $\operatorname{dim} M \geq 2$ If $\partial M \neq \phi)$.

(ii) a cartesian product of a countably infinite number of manifolds with nonempty boundary.

(iii) a cantor set.

Then orbital shadowing is generic property in $W$.

Acknowledgment. We would like to thank the referee for valuable suggestions.

\section{References}

[1] T. Choi, S. Kim, and K. Lee, Weak inverse shadowing and genericity, Bull. Korean Math. Soc. 43 (2006), no. 1, 43-52.

[2] B. A. Coomes, H. Koçak, and K. J. Palmer, Periodic shadowing, Chaotic numerics (Geelong, 1993), 115-130, Contemp. Math., 172, Amer. Math. Soc., Providence, RI, 1994.

[3] R. M. Corless and S. Yu. Pilyugin, Approximate and real trajectories for generic $d y$ namical systems, J. Math. Anal. Appl. 189 (1995), no. 2, 409-423.

[4] P. E. Kloeden and J. Ombach, Hyperbolic homeomorphisms and bishadowing, Ann. Polon. Math. 65 (1997), no. 2, 171-177.

[5] P. Koscielniak and M. Mazur, Chaos and the shadowing property, Topology Appl. 154 (2007), no. 13, 2553-2557.

[6] K. Kuratowski, Topology. Vol. II., Academic Press, New York-London; Państwowe Wydawnictwo Naukowe Polish Scientific Publishers, Warszawa, 1968.

[7] M. Mazur, Weak shadowing for discrete dynamical systems on nonsmooth manifolds, J. Math. Anal. Appl. 281 (2003), no. 2, 657-662.

[8] _ , Tolerance stability conjecture revisited, Topology Appl. 131 (2003), no. 1, 3338 .

[9] S. Yu. Pilyugin and O. B. Plamenevskaya, Shadowing is generic, Topology Appl. 97 (1999), no. 3, 253-266.

[10] F. Takens, On Zeeman's tolerance stability conjecture, Lecture Notes in Mathematics 197, Spriger-Verlag, pp. 209-219, 1971.

[11] P. Walters, On the pseudo-orbit tracing property and its relationship to stability, Lecture Notes in Math., 668, Springer, Berlin, pp. 231-244, 1978.

BaHMAN HonaRY

Department of Mathematics

FERDOWSI UNIVERSITY

MASHHAD 1159-91775, IRAN

E-mail address: honary@math.um.ac.ir

Alireza Zamani Bahabadi

Department of Mathematics

FERDOWSI UNIVERSITY

MASHHAD 1159-91775, IRAN

E-mail address: bahabadi@wali.um.ac.ir 